Cahiers $d u$ MONDE RUSSE

\section{Cahiers du monde russe}

Russie - Empire russe - Union soviétique et États indépendants

$59 / 4$ | 2018

Varia

\title{
Tracey A. Sowerby, Jan Hennings, Practices of Diplomacy in the Early Modern World c. 1410-1800
}

\section{Marie-Karine Schaub}

\section{OpenEdition}

\section{Journals}

Édition électronique

URL : https://journals.openedition.org/monderusse/10649

DOI : $10.4000 /$ monderusse. 10649

ISSN : $1777-5388$

\section{Éditeur}

Éditions de l'EHESS

\section{Édition imprimée}

Date de publication : 1 octobre 2018

Pagination : 619-622

ISBN : $978-2-7132-2747-9$

ISSN : $1252-6576$

Référence électronique

Marie-Karine Schaub, «Tracey A. Sowerby, Jan Hennings, Practices of Diplomacy in the Early Modern World c. 1410-1800 », Cahiers du monde russe [En ligne], 59/4 | 2018, mis en ligne le 01 octobre 2018, consulté le 06 janvier 2022. URL : http://journals.openedition.org/monderusse/10649 ; DOI : https:// doi.org/10.4000/monderusse.10649 
Tracey A. SOWERBY and Jan HENNINGS, eds.

\section{Practices of Diplomacy in the Early Modern World c. 1410-1800}

London - New-York : Routledge (Routledge Research in Early Modern History), 2017, 306 p.

L'ouvrage dirigé par Tracey A. Sowerby et Jan Hennings s'inscrit dans la suite de leurs travaux respectifs sur les pratiques diplomatiques en Europe à l'époque moderne, dans la monarchie Tudor pour la première et du côté russe pour le second. Ce livre collectif vient utilement enrichir une historiographie des relations internationales en constant renouvellement depuis une vingtaine d'années et confirmer l'inscription de la Russie sur la scène européenne dans la première modernité, en en faisant une monarchie comme une autre dans cette société des princes.

Après une solide et longue introduction programmatique par les deux directeurs de l'ouvrage (p. 1-21), le livre se compose de trois parties : la première prend en compte tout ce qui peut faire un « agent » diplomatique, la deuxième les dimensions sociales et culturelles des pratiques des acteurs diplomatiques et la troisième cherche à saisir la culture matérielle des échanges à travers les objets et les animaux. La première appelée « Statut et souveraineté au-delà de l'État » couvre des espaces comme la Bourgogne (p. 25-43), la Transylvanie (p. 44-64), Raguse (p. 65-79), mais aussi des moments pivots de rencontres diplomatiques, comme celles qui se tinrent à l'époque des négociations de la paix de Westphalie (p. 80-94). Dans une deuxième partie, ce sont les questions de la familiarité, des spectacles et des acteurs qui sont traitées, à travers l'hospitalité diplomatique à Gênes (p. 97-113), la naissance de l'opéra au XVII ${ }^{\mathrm{e}}$ siècle (p. 114-129), le rôle des ambassadrices et des femmes en général dans les échanges diplomatiques (p. 130-146), la figure des marchands-diplomates hollandais à la cour d'Aurangzeb (p. 147-165) ou celles des ambassadeurs ottomans à la cour de Vienne (p. 166-184). La troisième partie enfin, traite des « objets et des bêtes », généralement échangés comme cadeaux diplomatiques sous les Tudor et les Stuarts (p. 187-204), dans la péninsule ibérique à l'époque du règne de Ferdinand d'Aragon (p. 204-218) dans les relations entre Saffavides et Moghols au $\mathrm{XVII}^{\mathrm{e}}$ siècle (p. 219-236) ou encore dans celles entre l'Angleterre et la Moscovie à la fin du XVII ${ }^{\mathrm{e}}$ siècle (p. 237-253). L'ouvrage se termine par une conclusion de $\mathrm{Ch}$. Windler qui répond en miroir à certaines formulations de l'introduction. 
Il n'est pas étonnant que l'introduction commence par une description détaillée du carrosse envoyé par le roi Jacques $1^{\text {er }}$ en 1604 à Boris Godunov, alors tsar. L'objet symbolise l'amitié anglo-russe en utilisant l'héraldique des deux couronnes, un vocabulaire commun parfaitement compris par les deux parties. L'échange de cadeaux diplomatiques se place dans ce qui est au cœur de la démonstration des deux auteurs, à savoir que les pratiques sont à la base des relations internationales au début de l'époque moderne, qu'elles en apprennent plus sur leur nature au moment où leurs règles ne sont pas encore formalisées (p. 2). Dans sa conclusion, Ch. Windler rappelle, à juste titre, que le mot diplomatie n'apparaît pas avant la fin du XVIII ${ }^{\mathrm{e}}$ siècle, plus exactement en 1798 dans la $5^{\mathrm{e}}$ édition du Dictionnaire de l'Académie française : "Science des rapports, des intérêts de Puissance à Puissance » (p. 254). Il souligne donc le décalage entre cette définition qui renvoie à des compétences particulières voire à une science et la période chronologique ici adoptée, qui s'achève en 1800. Il signale également que l'histoire conceptuelle n'est pas très intégrée aux ouvrages traitant des questions diplomatiques, alors que les années 1800 sont celles de Sattelzeit qui, chez R. Koselleck, représentent une période charnière où le langage s'est transformé de langage prémoderne en langage moderne et où les relations entre les différents pouvoirs sont devenues des relations internationales, c'est-à-dire des relations entre des formes politiques organisées en nations à l'intérieur du cadre des États-nations et répondant à l'ordre international né des guerres napoléoniennes.

Lorsque les auteurs de l'ouvrage font référence à la diplomatie, ils renvoient à des pratiques politiques qui ne correspondent certainement pas à ce que vivaient les acteurs dont ils décrivent les comportements et les cadres mentaux (p. 254). Le terme même de diplomatie est donc ici un compromis dans une entreprise assumée par les deux directeurs de l'ouvrage non pas d'offrir une nouvelle définition de la diplomatie de la première modernité mais de problématiser le terme en le reliant à des pratiques qui ne sont pas nécessairement incluses dans les implications modernes du mot. Il faut, selon eux, dans l'étude de chaque rencontre, et si les sources le permettent, tenir de front le niveau politique des relations internationales, les questions de culture et de symbolique. Ils partent du constat que ce que l'on appelle " nouvelle histoire diplomatique » a ouvert des champs sur ce qui influence les relations diplomatiques, en mettant l'accent sur les individus et leur persona/leur Agency, en soulignant les tensions entre leurs intérêts personnels, la personne qu'ils représentent, leur entourage et leur clientèle ainsi que tous les intermédiaires qui alimentent ces relations. Ces approches conduisent à prendre moins en compte les questions de périodisation mais à s'intéresser à la continuité ou aux adaptations des processus et des pratiques ainsi qu'à l'intra et interculturel (p. 3). D'où l'importance de prendre en compte différents types de communications symboliques, le palais, les gestes ou les cadeaux (p. 4).

Parmi les multiples thèmes mis en lumière dans l'ouvrage, quatre émergent tout particulièrement : la souveraineté, les normes culturelles, le rôle des femmes et les différentes formes de langage mises en œuvre dans la diplomatie. 
On le sait bien, les cadres politiques de la souveraineté restent extrêmement indéfinis dans la première modernité. Et ce ne sont sûrement pas les structures qui permettent de délimiter de la manière la plus efficace l'espace de l'interaction politique, à moins de suivre $\mathrm{A}$. de Wicquefort lorsqu'il écrit dans L'ambassadeur et ses fonctions qu' "il n'y a point de plus illustre marque de la Souveraineté que le Droit d'envoyer \& de recevoir des Ambassadeurs » (cité p. 4). Travailler à partir des relations internationales permet donc d'interroger les contours de la souveraineté. Les développements qui concernent, par exemple, les relations entre le Saint Empire et l'Empire ottoman ou ceux sur la politique extérieure de la république de Raguse montrent le rôle majeur du prince de Transylvanie dans le premier cas (p. 44-47) et de la catégorie des diplomates-écrivains dans le second (p. 73-75). Ces deux cas montrent qu'il est indispensable de faire émerger des types d'acteurs à un niveau infra-étatique ou de n'omettre aucune strate politique pour saisir la complexité des interactions.

La question de la confrontation des normes culturelles est également au cœur de la démonstration de l'ouvrage. Les acteurs diplomatiques savent adapter leurs codes, en se comportant comme des acteurs locaux, en s'intégrant à des sociabilités locales et en acceptant des accommodements cérémoniels. Ils font preuve d'une très grande flexibilité et cette « perméabilité diplomatique » (p. 8) permet de faciliter les relations en jeu au-delà des expressions cérémonielles de la souveraineté et l'éclat des représentations aristocratiques. La rencontre culturelle et la négociation des normes sont au cœur de la relation diplomatique quel que soit le cadre géographique, très souvent tributaire d'un héritage eurocentrique. Même dans le cas de relations dominants/dominés, les représentants des «dominants » doivent s'adapter à la culture locale et opérer des négociations verbales et symboliques. Par exemple, les marchands de la VOC en Inde sont moins reconnus que les envoyés royaux, mais, moins pris aussi dans les ratiocinations cérémonielles, ils possèdent une plus grande marge de manœuvre pour négocier et maintenir des liens de l'intérieur (p. 153-157). La familiarité des acteurs avec les codes de leurs interlocuteurs est, la plupart du temps acquise lors des échanges, et elle renvoie à la question de la commensurabilité culturelle dans les relations que ce soit dans celles que mènent les agents de la VOC ou dans le cas des diplomates ottomans dans la sociabilité viennoise, qui finissent par montrer une «familiarité trans-impériale » à force de répétition (p. 166).

En ce qui concerne les femmes diplomates, les ambassadrices sont un exemple de la manière dont un groupe peut manipuler son rôle ou être utilisé lorsque les spécificités de celui qui doit mener la mission l'empêchent de la conduire à bon port. Elles sont souvent utilisées pour accéder à de l'information ou à des acteurs politiques. Les représentantes des familles royales sont, en raison de leur rang, intégrées aux réseaux de correspondances et d'échanges de cadeaux, de la même manière qu'elles sont somptueusement reçues. Dans le contexte de l'installation progressive d'une diplomatie permanente, un nombre grandissant de diplomates était accompagné de leurs épouses, considérées comme des actrices politiques importantes. Les carrières de Lady Elizabeth Trumbull et de Lady Mary Wortley 
Montagu montrent que les femmes pouvaient être un atout pour leur époux dans le cadre d'un « couple au travail » et pour réussir la mission de l'ambassade, en gérant les affaires domestiques et l'administration financière ou en organisant l'accueil des courriers et des autres ambassadeurs (p. 139). Prendre en considération la dimension genrée de la diplomatie permet de mettre en évidence le lien très fort entre la division sexuée des fonctions et la compétition pour les honneurs et les statuts. Certaines femmes pouvaient bénéficier d'un rituel diplomatique plus prestigieux qu'un courtisan masculin. Ici le rang est plus important que le genre et les frontières de genre y sont donc brouillées.

Enfin, le livre prend à bras-le-corps la question de la variété des types de langage, à la suite des travaux d'Isabella Lazzarini sur les stratégies discursives dans les interactions diplomatiques dans l'Italie renaissante. En effet, l'espace, la cérémonie ou la culture matérielle représentent des modes importants d'interaction politique dans les cours de l'Europe de la première modernité et ces langages sont utilisés pour exprimer la faveur ou reconnaître le statut (p. 14). Parmi ces modalités, le cadeau diplomatique illustre les voies complexes selon lesquelles les différents types de communication non verbale pouvaient entrer en relation avec la pratique diplomatique. Jan Hennings, consacre un chapitre à la question des cadeaux entre l'Angleterre et la Russie dans les années 1660 (p. 237-253). À travers l'étude du cadeau dont on sait qu'il est le lieu d'une formalisation accrue à l'époque moderne pour maintenir la fiction de la bonne volonté, tenter de prévenir les éventuels conflits et préserver l'équilibre de la réciprocité, ce chapitre met en lumière le fonctionnement des relations diplomatiques entre l'Angleterre et la Russie au XVII ${ }^{\mathrm{e}}$ siècle. Il étudie en effet, l'échec de deux ambassades, l'une à Londres en 1662 et l'autre à Moscou en 1663 et les stratégies utilisées par les ambassadeurs dans leurs acceptations et leurs refus des cadeaux offerts. L'article démontre que le maniement de ces objets est un relais du langage politique ou sert d'appui aux négociations. Parfois, leur échange permet de compenser des dysfonctionnements cérémoniels et parfois ils échouent. C'est le cas lorsque Carlisle, l'ambassadeur anglais, voulant restaurer l'honneur de son roi qu'il estime bafoué lors d'un dysfonctionnement cérémoniel met en œuvre une véritable « contre-offensive rituelle » et refuse les cadeaux qui lui sont offerts (p. 245-246). Malgré l'échec formel de l'ambassade, le jeu sur les cadeaux montre un système commun de valeurs et de normes culturelles partagées et non l'éviction de la Russie du cercle des États chrétiens. Même si Carlisle a exclu la Russie de la scène européenne à son retour en Angleterre pour justifier l'échec de son ambassade et que cette stratégie discursive a été reprise, d'abord par A. de Wicquefort et ensuite par Voltaire, l'affaire de ces cadeaux signale une maîtrise parfaite d'une grammaire de l'échange de part et d'autre (p. 247).

Ce livre est un exemple important du renouvellement de l'histoire des relations internationales, dont la lecture est nécessaire pour saisir les structures politiques de la première modernité européenne, Russie incluse. 